\title{
A Perspetiva da Toxicologia Clínica Sobre a Utilização Terapêutica da Cannabis e dos Canabinoides
}

\section{The Clinical Toxicology Perspective on the Therapeutic Use of Cannabis and Cannabinoids}

Ricardo Jorge DINIS-OLIVEIRA $\triangle^{1,2,3}$

Acta Med Port 2019 Feb;32(2):87-90 - https://doi.org/10.20344/amp.10896

Palavras-chave: Canabinoides/uso terapêutico; Cannabis/uso terapêutico; Marijuana Medicinal/efeitos adversos

Keywords: Cannabinoids/therapeutic use Cannabis/therapeutic use; Medical Marijuana/adverse effects

\section{INTRODUÇÃO}

Os derivados da planta cannabis sativa são as substâncias psicoativas recreativas ilícitas de maior consumo mundial. ${ }^{1,2}$ Dos mais de 100 canabinoides já descritos, os fitocanabinoides delta-9-tetrahidrocanabinol ( $\Delta^{9}$-THC; principal constituinte psicoativo), $\Delta^{8}$-THC (quase tão ativo como o anterior, mas em menores concentrações), canabinol (pouco ativo, mas em elevadas concentrações) e o canabidiol (não psicoativo mas presente em elevadas concentrações na planta), são aqueles que melhor se conhecem do ponto de vista científico ${ }^{3,4}$ Destes, o $\Delta^{9}$-THC e o canabidiol têm eficácia farmacológica comprovada e as suas concentrações na planta são diferentes entre o caule, folhas ou flores e altamente dependentes do teor de humidade, luz e da tipologia do solo., ${ }^{3,4} \mathrm{O}$ conhecimento sobre o funcionamento do sistema dos endocanabinoides endógenos em vários processos fisiológicos (e.g., memória, ansiedade, imunidade, dor, regulação do apetite, etc.) mostra como estes podem ter um efeito terapêutico em algumas patologias e também que a sua ausência endógena poderá explicar a patofisiologia de algumas doenças prevalentes. ${ }^{5} \mathrm{~A}$ natureza ensina-nos, pois, se não fossem importantes, não teríamos recetores - muito provavelmente endógenos - para os canabinoides nomeadamente em neurónios (recetores $\mathrm{CB}_{1}$ ) e em várias células do sistema imunológico (recetores $\mathrm{CB}_{2}$ ). ${ }^{6}$ Numa pesquisa em bases de dados científicas na área da medicina (credíveis e alvo de processo de revisão pelos parceiros científicos) utilizando as palavras-chave cannabinoids, therapeutic use e clinical trials, fica claro que os fitocanabinoides estão já licenciados em vários países para a terapêutica de várias patologias, algumas com forte evidência científica, e outras em que a evidência não é tão óbvia, mas mesmo assim promissora. ${ }^{7-9} \mathrm{O}$ tratamento das náuseas e vómitos associados à quimioterapia, como analgésicos na dor crónica, na anorexia associada à síndrome de imunodeficiência adquirida humana, dor neuropática e da espasticidade em algumas doenças neurológicas como a esclerose múltipla, ansiedade, insónia e epilepsia na idade pediátrica, doença de Parkinson, sobretudo nas formas refratárias à terapêutica convencional, são algumas das indicações terapêuticas mais documentadas e que justificam alterações do enquadramento legal para uma regulação da sua aplicação terapêutica. ${ }^{10,11}$ Estão descritos ainda vários outros estudos que mostram potencial na utilização destes fármacos como antiespasmódicos, neuroprotetores, imunomoduladores, na doença de Alzheimer, na fibromialgia, na apneia obstrutiva do sono e também em algumas doenças oncológicas como anticancerígenos. ${ }^{12-14}$

Neste artigo pretende-se discutir a perspetiva do toxicologista e da toxicologia clínica e forense da utilização terapêutica da Cannabis medicinal e dos fitocanabinoides puros.

\section{Questões em aberto: o que é mito?}

A inquietude da toxicologia não é pois se os fitocanabinoides têm ou não atividade terapêutica, porque essa está mais que demonstrada. A questão também não é se o seu poder psicoativo é ou não muito elevado, pois não o é definitivamente em termos absolutos, mas também em termos comparativos com outras substâncias psicoativas. ${ }^{3,6}$ Na verdade muitas das substâncias psicoativas em comercialização apresentam um poder aditivo bem mais elevado que o $\Delta^{9}$-THC como é o caso do etanol, que é lícito, e outras, como o caso da morfina, que está disponível como fármaco analgésico e para outras indicações clínicas. ${ }^{3,6}$ A dependência, doença neuropsiquiátrica, desenvolve-se mais facilmente com substâncias psicoativas que produzem um intenso prazer (euforia e felicidade), ou seja, recompensa. ${ }^{15} \mathrm{~A}$ dopamina é o neurotransmissor implicado na dependência/adição às substâncias psicoativas sendo que as substâncias classificadas de grau 1 , são consideradas não aditivas, as de grau 2 (como $\circ \Delta^{9}$-THC) são as de mais baixo poder aditivo, seguindo-se as de grau 3 (e.g., etanol), 4 (e.g., vários opioides) e 5 (e.g., cocaína). ${ }^{16-18}$ Mesmo assim, apenas uma pequena percentagem dos doentes desenvolvem dependência, como são exemplo aqueles em terapêutica analgésica com opioides. ${ }^{19}$

1. Department of Public Health and Forensic Sciences, and Medical Education. Faculty of Medicine. University of Porto. Porto. Portugal.

2. Institute of Research and Advanced Training in Health Sciences and Technologies. Department of Sciences. University Institute of Health Sciences. Gandra. Portugal.

3. Laboratory of Toxicology. Department of Biological Sciences. Faculty of Pharmacy. University of Porto. Porto. Portugal.

$\square$ Autor correspondente: Ricardo Jorge Dinis-Oliveira. ricardinis@sapo.pt

Recebido: 03 de junho de 2018 - Aceite: 10 de dezembro de 2018| Copyright @ Ordem dos Médicos 2019 
A toxicologia também não aceita que esta seja uma questão da exclusiva intervenção da política, mas sim de base e natureza científica. Como em outros fóruns tem sido referido, deverá respeitar-se o princípio que, "à ciência o que é da ciência, à política o que é da política”, não se devendo aprovar fármacos pelo bypassing de critérios científicos rigorosos.

A questão também não passa por vender a ideia de que a cannabis é o elixir da vida eterna ou a cura para todos os males, porque isso certamente não o será, mesmo que muitos a queiram vincular a todas, e a mais algumas, aplicações terapêuticas como se se tratasse de uma Caixa de Pandora. ${ }^{20,21}$

\section{Questões em aberto: o que não é mito?}

A questão é sim, de outra ordem, nomeadamente se devemos continuar com o estigma social sem discutir seriamente a importância da cannabis com critérios científicos. Dizer que temos aprovados no mercado os canabinoides $\Delta^{9}$-THC (sob a designação de dronabinol na sua forma sintética) e canabidiol, e portanto assumir que já existem na terapêutica não é adequado uma vez que o número de utilizadores e de prescritores é muito reduzido.

$\mathrm{Na}$ verdade, segundo dados da Autoridade Nacional do Medicamento e Produtos de Saúde, entre os anos 2016 e 2017 foram prescritas 21 unidades do fármaco Sativex ${ }^{\circledR}$ (e.g., "nabiximols", contém uma razão de $\Delta^{9}$-THC/canabidiol) e todas pelo Centro Hospitalar de Lisboa Central. Em Portugal temos já há vários anos plantações autorizadas (duas de cannabis e duas da papoila do ópio) para o cultivo e exportação para multinacionais da indústria farmacêutica (e.g., Aviso n. ${ }^{\circ}$ 10618/2014 de 25 de Agosto publicado em

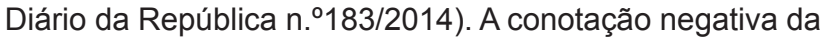
cannabis retrai e limita a sua investigação e a utilização legítima dos seus constituintes para fins terapêuticos. É de valorizar o debate sério em que se tem envolvido a comunidade científica, para além da sociedade civil e política. ${ }^{22,23}$

A questão passa por colher evidências empíricas a partir de publicações científicas e também de outros países que já utilizam a cannabis para fins recreativos e/ou terapêuticos, como o Canadá, a Alemanha, a Holanda, a Itália e os Estados Unidos e utilizá-las como suporte científico da jurisprudência para legitimar ou não a utilização terapêutica destas substâncias. Recentemente o Canadá, através do "Cannabis Act" de 21 de junho de 2018, legalizou o consumo de cannabis para fins recreativos à semelhança de outras latitudes como o Uruguai e em vários estados Norte-Americanos. Esta é, todavia, uma opção política e que não será aqui explorada. Paralelamente, o Niagara College de Ontário, no Canadá, começou recentemente a lecionar um curso de "Produção Comercial de Cannabis" para fins terapêuticos com a indicação de que "there is a new and overwhelming need for qualified and trained professionals in the booming cannabis production industry".

A questão passa também por uma legalização regulamentada de toda a cadeia de produção industrial da cannabis, distribuição e consumo, rigoroso controlo de qualidade do produto final, registos dos consumidores, definição de idades autorizadas (como, por exemplo, a legislação sobre o etanol que proíbe a sua venda a menores de 18 anos conforme definido pelo Decreto-Lei n. ${ }^{\circ}$ 106/2015, de 16 de junho, continuação da proibição da condução rodoviária sob a influência destas substâncias como previsto em legislação própria, ${ }^{24}$ venda em farmácias com intervenção dos farmacêuticos na rede dos cuidados de saúde, e vigilância dos padrões de consumo (como aliás é feito para muitas outras substâncias) e proibição de publicidade e inclusão de mensagens de advertência para os efeitos secundários e adversos à semelhança do que acontece com o tabaco, entre outras medidas de natureza legislativa.

A questão passa também pela prescrição em receita especial, pela óbvia supervisão médica, por exemplo da Psiquiatria, sobretudo se da utilização resultarem psicoses e outras patologias de natureza psiquiátrica e pela educação e promoção do uso racional e responsável dos medicamentos. ${ }^{25-27}$

A questão pode ainda passar pela regulação do preço, para valores próximos dos do mercado ilícito, e aplicar os lucros da receita fiscal em políticas de educação para a saúde, no tratamento dos distúrbios dos consumos de substâncias psicoativas, e em estratégias forenses que permitam mitigar o mercado ilícito e a criminalidade e as redes organizadas de tráfico.

A utilização terapêutica dos princípios ativos da cannabis, $\Delta^{9}$-THC e canabidiol, é uma verdade incontestável e já alvo dos mais sérios estudos pré-clínicos e clínicos. ${ }^{5,7-9}$

\section{Propostas de resolução}

Recomendar a utilização terapêutica da cannabis, apesar de menos óbvia, porque a extrapolação dos resultados obtidos com os fitocanabinoides puros para a planta não é direta, é ainda assim defensável do ponto de vista científico, apesar do distinto nível de evidência para diferentes patologias e como tal são recomendáveis mais estudos. .,7-9 $^{-1}$ A verdade é que, mesmo depois da entrada em comercialização de qualquer substância para fins terapêuticos, os estudos toxicológicos continuam e até se intensificam. Já em 2018 um estudo ${ }^{28}$ concluiu que há evidência para apoiar o efeito terapêutico de cannabis e dos fitocanabinoides em várias patologias, sobretudo em condições refratárias a outras terapêuticas. Suportados nestes e noutros resultados, o Parlamento Português aprovou pela Lei n. ${ }^{\circ}$ 33/2018 de 18 de julho a utilização de cannabis para fins medicinais, apenas quando das terapêuticas convencionais não produzam os efeitos desejados, e apenas mediante a "sua prescrição e a dispensa em farmácia”. A partir de fevereiro de 2019, os médicos passaram a poder prescrever derivados da cannabis para determinadas patologias listadas pela Autoridade Nacional do Medicamento e Produtos de Saúde, I.P. (INFARMED).

Defender o auto-cultivo para fins medicinais, mesmo preconizando/contemplando a autorização prévia das autoridades de saúde, não será o caminho certo a percorrer, pois não é assim que se promove a utilização segura 
e monitorizada de qualquer medicamento, uma vez que da produção "caseira" resulta a heterogeneidade dos lotes e das concentrações dos canabinoides presentes, e, como tal, um imprevisível impacto toxicológico, farmacológico e na saúde pública. No fundo, seria um fator adicional de entropia a acrescentar à variabilidade da resposta que já de si é complexa por questões relacionadas com polimorfismos genéticos, dieta, patologias concomitantes, e muitos outros fatores. $^{4}$

É importante desmistificar a ideia segundo a qual só se administra cannabis se for fumada. Esta via de administração acarreta riscos toxicológicos como é o caso do cancro do pulmão. ${ }^{29}$ Seria, por isso, no mínimo muito atípico recomendarmos fumar, quando dispomos de medicamentos com os seus princípios ativos ou vias de administração da cannabis mais seguras. A cannabis pode ser administrada por via oral, na forma de infusões, gotas sublinguais, por via transdérmica, em vaporizações, etc. ${ }^{30-32}$ Existem muitos outros chás com muito menos evidências científicas que suportem a sua recomendação, e que mesmo assim são passíveis de aquisição (e.g., em farmácias) e amplamente consumidos.

A questão também não pode ser a Lei do tudo ou nada, como se estivéssemos à espera de demonstrar a inocuidade ou a plena segurança da cannabis. A farmacologia não se sustenta em crenças, e como tal, afirmar que a cannabis tem efeitos secundários ou adversos, é também uma verdade incontestável. Qual o composto farmacológico que deles está isento? Basta abrir um folheto informativo de um qualquer medicamento. No caso em concreto da cannabis, a maioria dos estudos parecem excluir de forma consistente efeitos tóxicos graves, sendo todavia de assinalar a congestão ocular, ataques de pânico, alterações imunológicas, dependência, síndrome de abstinência, tolerância, alucinações, ideação paranoide, alterações sensoriais, alterações cognitivas e visuais e do tempo de reação, sinestesias, e quadros de psicose tóxica, sobretudo se existirem antecedentes de esquizofrenia, entre outros efeitos. ${ }^{15}$

\section{CONCLUSÃO}

\section{Perspetivas Futuras}

Para além dos fitocanabinoides e dos endocanabinoides, nos últimos anos tem-se assistido à introdução de novas substâncias psicoativas, como é o caso dos cana- binoides sintéticos usados com fins recreativos, e que são muitas vezes mais potentes do que os presentes na planta e podem apresentar efeitos tóxicos imprevisíveis, inclusive fatais. ${ }^{13,33,34}$ Enquanto nestes derivados a realidade é sombria e de repercussões clínicas e forenses negativas, ${ }^{35}$ os fitocanabinoides têm já várias ações terapêuticas comprovadas cientificamente. Em ciência vivemos do apoio da estatística para suportar as nossas evidências. A estatística diz também que temos milhões de consumidores de cannabis. Estarão todos errados? Por exemplo no último relatório do Serviço de Intervenção nos Comportamentos Aditivos e nas Dependências (SICAD) em matéria de consumo matéria de drogas e toxicodependências a cannabis foi a substância psicoativa ilícita que registou as maiores prevalências de consumo (10\% ao longo da vida, $5 \%$ nos últimos 12 meses e $4 \%$ nos últimos 30 dias). Fomos pioneiros e elogiados com a descriminalização da posse de substâncias psicoativas para consumo próprio, passando a considerar o portador de distúrbio do uso de substâncias psicoativas (i.e., o toxicodependente) como doente e não um criminoso. Temos também agora margem para utilizar a cannabis para fins terapêuticos através da monitorização pelas entidades responsáveis, à semelhança do que é praticado em todos os medicamentos. O preconceito não faz sentido, a regulação sim e o futuro dir-nos-á se outras substâncias presentes na cannabis não poderão elas vir a ser também promissoras moléculas terapêuticas, como vários estudos já antecipam. ${ }^{36,37}$ Por fim, importa estarmos também atentos ao desenvolvimento de outras aplicações da cannabis, nomeadamente na indústria da cosmética (e.g., através da incorporação em cremes e géis), alimentar e têxtil. ${ }^{38,39}$

A cannabis está longe de ser o demónio da toxicologia. A Lei de Paracelso é clara: "é a dose que faz o veneno e é a dose que distingue o veneno do remédio". Por fim é importante reforçar a necessidade de se realizarem mais ensaios clínicos, notar a diferença que existe entre a cannabis medicinal como produto de composição complexa e os fitocanabinoides puros e a grande variabilidade interindividual em termos farmacocinéticos e farmacodinâmicos.

\section{CONFLITOS DE INTERESSE}

O autor declar não ter qualquer conflito de interesse relativamente ao presente artigo.

\section{REFERÊNCIAS}

1. United Nations Office on Drugs and Crime. World Drug Report 2017 executive summary conclusions and policy implications. Vienna: United Nations; 2017.

2. European Monitoring Centre for Drugs and Drug Addiction. European Drug Report 2017 - trends and developments. Lisbon: European Monitoring Centre for Drugs and Drug Addiction; 2017.

3. Dinis-Oliveira RJ. Usos lícito e ilícito dos fármacos. Acta Med Port. 2014;27:755-66.

4. Dinis-Oliveira RJ. Metabolomics of Delta9-tetrahydrocannabinol: implications in toxicity. Drug Metab Rev. 2016;48:80-7.

5. Katzung BG, Trevor AJ, editors. Basic \& clinical pharmacology. $13^{\mathrm{a}}$ ed. New York: McGraw-Hill; 2015.
6. Dinis-Oliveira RJ, Carvalho F. Farmacodinâmica das drogas e do álcool. In: Sexo, drogas e cérebro. Fardilha M, Soares A, editores. Porto: Afrontamento; 2016. p. 147-73.

7. Maccarrone $\mathrm{M}$, Maldonado $\mathrm{R}$, Casas $\mathrm{M}$, Henze $\mathrm{T}$, Centonze $\mathrm{D}$. Cannabinoids therapeutic use: what is our current understanding following the introduction of THC, THC: CBD oromucosal spray and others? Expert Rev Clin Pharmacol. 2017;10:443-55.

8. Noel C. Evidence for the use of "medical marijuana" in psychiatric and neurologic disorders. Ment Health Clin. 2017;7:29-38.

9. Fitzcharles MA, Eisenberg E. Medical cannabis: a forward vision for the clinician. Eur J Pain. 2018;22:485-91.

10. Rong C, Lee Y, Carmona NE, Cha DS, Ragguett RM, Rosenblat JD, 
et al. Cannabidiol in medical marijuana: research vistas and potential opportunities. Pharmacol Res. 2017;121:213-8.

11. Mucke M, Phillips T, Radbruch L, Petzke F, Hauser W. Cannabis-based medicines for chronic neuropathic pain in adults. Cochrane Database Syst Rev. 2018;3:CD012182.

12. Birdsall SM, Birdsall TC, Tims LA. The use of medical marijuana in cancer. Curr Oncol Rep. 2016;18:40.

13. Mastinu A, Premoli M, Ferrari-Toninelli G, Tambaro S, Maccarinelli G, Memo M, et al. Cannabinoids in health and disease: pharmacological potential in metabolic syndrome and neuroinflammation. Horm Mol Biol Clin Investig. 2018. [Epub ahead of print]

14. Solimini R, Rotolo MC, Pichini S, Pacifici R. Neurological disorders in medical use of cannabis: an update. CNS Neurol Disord Drug Targets. 2017; 16:527-33.

15. Dinis-Oliveira RJ, Carvalho F, Bastos ML. Toxicologia forense. Lisboa: Lidel, Edições Técnicas LDA; 2015.

16. Walker DM, Nestler EJ. Neuroepigenetics and addiction. Handb Clin Neurol. 2018;148:747-65.

17. Chen W, Nong Z, Li Y, Huang J, Chen C, Huang L. Role of dopamine signaling in drug addiction. Curr Top Med Chem. 2017;17:2440-55.

18. Becker JB, Chartoff $E$. Sex differences in neural mechanisms mediating reward and addiction. Neuropsychopharmacology. 2019;44:166-183.

19. Johnson BA. Addiction medicine: science and practice. London: Springer; 2011.

20. Solymosi K, Kofalvi A. Cannabis: a Treasure Trove or Pandora's Box? Mini Rev Med Chem. 2017;17:1223-91.

21. Lucas CJ, Galettis P, Schneider J. The pharmacokinetics and the pharmacodynamics of cannabinoids. Br J Clin Pharmacol. 2018;84:247782.

22. Clark PA, Capuzzi K, Fick C. Medical marijuana: medical necessity versus political agenda. Med Sci Monit. 2011;17:Ra249-61.

23. Sachs J, McGlade E, Yurgelun-Todd D. Safety and toxicology of cannabinoids. Neurotherapeutics. 2015;12:735-46.

24. Dinis-Oliveira RJ, Nunes R, Carvalho F, Santos A, Teixeira H, Vieira $\mathrm{DN}$, et al. Procedimentos técnicos, éticos e legais da competência do médico no cumprimento da lei da fiscalização da condução rodoviária sob influência do álcool e substâncias psicotrópicas. Acta Med Port. 2010;23:1059-82.

25. Carter GT, Weydt P, Kyashna-Tocha M, Abrams DI. Medicinal cannabis: rational guidelines for dosing. IDrugs. 2004;7:464-70.

26. Banys P, Cermak TL. Marijuana legalization in California: rational implementation of the adult use of marijuana Act (AUMA). J Psychoactive Drugs. 2016;48:63-5.

27. Jaffee WB, D'Zurilla TJ. Personality, problem solving, and adolescent substance use. Behav Ther. 2009;40:93-101.

28. Abrams DI. The therapeutic effects of Cannabis and cannabinoids: An update from the National Academies of Sciences, Engineering and Medicine report. Eur J Intern Med. 2018;49:7-11.

29. Callaghan RC, Allebeck P, Sidorchuk A. Marijuana use and risk of lung cancer: a 40-year cohort study. Cancer Causes Control. 2013;24:181120.

30. Dryburgh LM, Bolan NS, Grof CP, Galettis P, Schneider J, Lucas CJ, et al. Cannabis contaminants: sources, distribution, human toxicity and pharmacologic effects. Br J Clin Pharmacol. 2018;84:2468-76.

31. Shiplo S, Asbridge M, Leatherdale ST, Hammond D. Medical cannabis use in Canada: vapourization and modes of delivery. Harm Reduct J. 2016;13:30.

32. Russell C, Rueda S, Room R, Tyndall M, Fischer B. Routes of administration for cannabis use - basic prevalence and related health outcomes: a scoping review and synthesis. Int J Drug Policy. 2018;52:8796.

33. Cohen K, Weinstein A. The effects of cannabinoids on executive functions: evidence from cannabis and synthetic cannabinoids-a systematic review. Brain Sci. 2018;8.

34. Sud P, Gordon M, Tortora L, Stripp M, Borg D, Berman A. Retrospective chart review of synthetic cannabinoid intoxication with toxicologic analysis. West J Emerg Med. 2018;19:567-72.

35. Paul AB, Simms L, Amini S, Paul AE. Teens and spice: a review of adolescent fatalities associated with synthetic cannabinoid use. J Forensic Sci. 2018;63:1321-4.

36. Noreen N, Muhammad F, Akhtar B, Azam F, Anwar MI. Is cannabidiol a promising substance for new drug development? A review of its potential therapeutic applications. Crit Rev Eukaryot Gene Expr. 2018;28:73-86.

37. Maule WJ. Medical uses of marijuana (Cannabis sativa): fact or fallacy? Br J Biomed Sci. 2015;72:85-91.

38. Eagleston LR, Kalani NK, Patel RR, Flaten HK, Dunnick CA, Dellavalle RP. Cannabinoids in dermatology: a scoping review. Dermatol Online J. 2018;24.

39. Frassinetti S, Moccia E, Caltavuturo L, Gabriele M, Longo V, Bellani L, et al. Nutraceutical potential of hemp (Cannabis sativa L.) seeds and sprouts. Food Chem. 2018;262:56-66. 